\title{
Análisis de indicadores de desarrollo sostenible en el municipio de São João da Ponta/PA
}

Los estudios sobre Índices de Desarrollo Sostenible Municipales (IDSM) han sido muy importantes para comprender las disparidades existentes entre municipio de una misma región. Algunas metodologías de construcción de índices tienen su origen en el análisis de variables y datos obtenidos de institutos oficiales de investigación. Estos constituyen los indicadores fundamentales para los estudios integrados El objetivo de este artículo es presentar el IDSM de un municipio de norte de Brasil, considerando indicadores multidimensionales. El municipio de São João da Ponta está ubicado en la zona costera del Estado de Pará, en la región norte de Brasil. Tiene una superficie de $195.918 \mathrm{~km}^{2}$, una población estimada de 6.139 habitantes y una densidad demográfica de $26,87 \mathrm{hab} / \mathrm{km}^{2}$. Para este estudio se consideraron tres dimensiones: Social, Económica y Ambiental, las cuales se trabajaron con los datos disponibles en las plataformas virtuales de las agencias, instituciones del Gobierno Federal para realizar el análisis. Finalmente, se encontró que el municipio de São João da Ponta tiene un estado de alerta con un indicador social de 0.44; para la dimensión económica, se encuentra en estado crítico, con 0,19; y en estado de alerta, con 0,43, para la dimensión ambiental. El resultado de la relación entre las tres dimensiones, denominado 'Triple Bottom Line', tuvo un valor de 0,35, lo que pone en alerta al municipio. Se espera que el estudio pueda contribuir a la toma de decisiones por parte de la gestión municipal.

Palabras clave: Desarrollo Sostenible; Indicadores Multidimensionales; São João da Ponta.

\section{Análise dos indicadores de desenvolvimento sustentável do municipio de São João da Ponta/PA}

Os estudos sobre Índices de Desenvolvimento Sustentável Municipal (IDSM) têm sido muito importante para compreender as disparidades existentes entre os municípios de uma mesma região. Algumas metodologias de construção de Índices tem origem na análise de variáveis e dados obtidos nos institutos de pesquisas oficiais. Esses compõem os indicadores fundamentais para estudos integrados. O objetivo desse artigo é apresentar o IDSM de um município da região Norte do Brasil, considerando indicadores multidimensionais. O município de São João da Ponta localiza-se na zona costeira do Estado do Pará, região Norte do Brasil. Possu uma área de $195.918 \mathrm{~km}^{2}$, população estimada em 6.139 habitantes e densidade demográfica de $26,87 \mathrm{hab} / \mathrm{km}^{2}$. Para este estudo, foram consideradas três dimensões: Social, Econômica e Ambiental, as quais foram trabalhadas com os dados disponíveis nas plataformas virtuais dos órgãos, instituições do Governo Federal para a realização da análise. Por fim, verificou-se que o município de São João da Ponta possui estado de alerta com indicador social de 0,$44 ;$ para a dimensão econômica, apresenta-se em estado crítico, com 0,19; e em estado de alerta, com 0,43, para a dimensão ambiental. 0 resultado da relação entre as três dimensões, chamada de 'Triple Bottom Line' teve como valor 0,35, que coloca o município em estado de alerta. Espera-se que o estudo possa contribuir para tomada de decisão pela gestão municipal.

Palavras-chave: Desenvolvimento Sustentável; Indicadores Multidimensionais; São João da Ponta.

Topic: Geografia Regional

Reviewed anonymously in the process of blind peer.
Received: 02/12/2020

Approved: 22/02/2021
Christian Dávila Pinedo (it)

Universidade Federal do Pará, Brasil http://lattes.cnpq.br/9520191720018177

http://orcid.org/0000-0001-6430-2604

christiandavilapinedo@gmail.com

Marcia Aparecida da Silva Pimentel

Universidade Federal do Pará, Brasil

http://lattes.cnpq.br/3994635795557609

http://orcid.org/0000-0001-9893-9777

mapimentel@ufpa.br
Referencing this:

PINEDO, C. D.; PIMENTEL, M. A. S.. Análisis de indicadores de desarrollo sostenible en el municipio de São João da Ponta/PA. Nature and Conservation, v.14, n.1, p.158-168, 2021. DOI: http://doi.org/10.6008/CBPC2318-2881.2021.001.0018 


\section{INTRODUCCIÓN}

Actualmente las sociedades al nivel mundial están preocupados para la preservación de sus recursos naturales, que son la principal materia prima y riqueza de cada país; por eso tienen en cuenta el Desarrollo Sostenible como tema central en sus debates, controversias, políticas, etc. para llegar a una o muchas soluciones para el problema de la escases de los RRNN tanto en el sector Público como privado (CASTILLO et al., 2017).

La responsabilidad ambiental es para todos, porque sin el accionar del ser humano, principal actor causante de las variaciones en el medio ambiente gracias con la aparición de la Revolución Industrial en lo que al principio no se tuvo en cuenta al medio ambiente y solo era el objetivo de producir y ganar dinero; no se pueden tener algunos cambios y mejoras en el proceso del desarrollo sostenible (CASTILLO et al., 2017)

Siendo, que en el Informe de "Our Common future" o informe de Brundtland, por primera vez fue definida el concepto de desarrollo sostenible como el que responde a las necesidades del presente en forma igualitaria sin comprometer las necesidades del futuro (CASTILLO et al., 2017).

Referente a la evaluación de la sustentabilidad de un lugar, es un poco complicado porque no hay una ecuación o formula exacta para aplicarlos en todos de la misma forma; pues eso quiere decir que hay varias instancias en la cual se debe de tomar en cuenta por que cada sitio tiene su peculiaridad, características propias para la toma de decisiones en los sectores privados como públicos. Para eso están los indicadores para dar una dirección a los evaluadores, pueden estar inmersos a varias escalas al nivel local, regional y hasta nacional, depende la investigación que se esté realizando para donde va su visión y ver el grado de desarrollo sostenible que resulte (CRISPIM et al., 2019).

De acuerdo con el IDH (ÍNDICE de desarrollo humano), que es un indicador que refleja el nivel de desarrollo de cada país; muestra al Brasil en el puesto 79으 con un 0.761 de valor, teniendo como primer lugar a Noruega seguida de Suiza con 0.954 y 0.946 respectivamente (PNUD, 2019).

\section{DISCUSIÓN TEÓRICA}

\section{Dimensión social}

La idea de la participación general de todos los actores involucrados de la comunidad en la toma de decisiones, respetando opiniones y/o aportes de los otros. Donde la el color de piel, raza, genero, religión, etc. es un punto relevante ya que se tiene como objetivo el trabajo en equipo y en la solución de conflictos (PÉREZ, 2005).

También, son incluidos su demografía de la población, vivienda, alimentación, salud, etc. que tienen que ver directamente con los RRHH importantes para el proceso de desarrollo sostenible de un determinado lugar (GARAY, 2020).

Los individuos y los factores sociales trabajando en conjunto, con la ayuda de técnicas, metodologías y conocimientos locales, se tendrán buenos resultados como el crecimiento de una sociedad más unida y transparente y en la mejora de la calidad de vida de sus habitantes (GÁMEZ, 2014). 


\section{Dimensión económica}

En esta dimensión se tiene una preocupación en la cual un país tenga el crecimiento económico racional y bien gestionado, agregando a ello los ingresos y egresos, todo lo que tiene que ver con el dinero; pero trabajando de la mano con los temas sociales y el medio ambiente; o sea, que la sociedad y la naturaleza puedan estar presente, porque no es solo generar dinero, es también cuidar de los recursos naturales y humanos, porque gracias a ello una sociedad tiene su crecimiento (PÉREZ, 2005).

En la integración de las herramientas y materia prima, presentes en la vida cotidiano de una comunidad rural para poder usarla sabiamente y tener buenos resultados como los ingresos y el crecimiento económico de los mismos, que es muy importante para los pobladores (GARAY, 2020)

\section{Dimensión ambiental}

En esta dimensión también es conocido como Dimensión ambiental, donde se tiene que trabajar en la producción de la materia prima, pero respetando o cumpliendo con las leyes de la naturaleza, procesos físicos y químicos de ellos, sin comprometer a las futuras generaciones; en ella también se puede decir que los propios pobladores deben tener una cultura ambientalista (PÉREZ, 2005).

Además, es generar en el sujeto una mentalidad en la que tiene que vivir en armonía con la naturaleza, y sobre todo que gracias a esta interacción se pueda llegar al desarrollo sostenible, un hecho claro es en el respeto a la cultura y a las tradiciones que tienen que ver con la naturaleza (GARAY, 2020)

Parte de esta armonía, se encuentran los manglares pertenecen a los ecosistemas de humedales que se encuentran en América Latina, están presentes en 150 países y es internacionalmente valorado por la presencia de micro climas, recursos naturales de fauna y flora propias de los humedales (KOTZIAN et al., 2004), por lo que São João da Ponta Cuenta con su propio humedal pero otorgado por ell gobierno brasilero como una Reserva de Extracción de sus recursos marinos de uso sostenible (; sin embargo, existen el lado negativo d la historia, en la que hay personas que entran al lugar sin ningún permiso a depredar dichas áreas, por lo que los poblados, que son participes en el desarrollo de la Resex, realizan vigilancia constante.

\section{METODOLOGÍA}

El municipio de São João da Ponta, pertenece al sistema Costero Marino, dentro del Estado de Para, posee un área de 195,918 km², cuenta con una población estimada de 6.139 personas con una densidad demográfica de $26,87 \mathrm{hab} / \mathrm{km}^{2}$, hace frontera con los municipios de São Caetano de Odivelas y Santo Antônio do Tauá (IBGE, 2010).

La ubicación central del municipio es en la latitud $00^{\circ} 50^{\prime} 59^{\prime \prime}$ sur y la longitud $47^{\circ} 55^{\prime} 12^{\prime \prime}$ Oeste, con una altitud de 34 metros sobre el nivel del mar. Sus límites son al norte y oeste con São Caetano de Odivelas, al sur con los municipios de Terra Alta y São Caetano de Odivelas, y al este con los municipios de Terra Alta y Curuçá (Figura 1). El acceso es por la carretera PA-136 (Rodovia Castanhal - Curuçá) y PA-3759 (Lei Estadual no 592 de 27 de diciembre del 1995) 
El municipio de São Joao da Ponta cuenta con un Índice de Desarrollo Humano Municipal (IDHM) de 0,583 (IBGE, 2010), lo cual se posiciona como el municipio con bajo IDHM, la longevidad, ingresos y educación son los sectores que dan más resultados con 0,767, 0,522 y 0,495 respectivamente, en 2010 (BRASIL, 2020).

El salario mensual promedio fue de 1,6 salarios mínimos. La proporción de ocupados en relación con la población total fue del 5,2\%. En comparación con los demás municipios del estado, fue de 126 en 144 y 113 en 144, respectivamente. En comparación con las ciudades de todo el país, ocupó el puesto 4.427 con 5.570 y 5.175 con 5.570 , respectivamente., en el 2018.

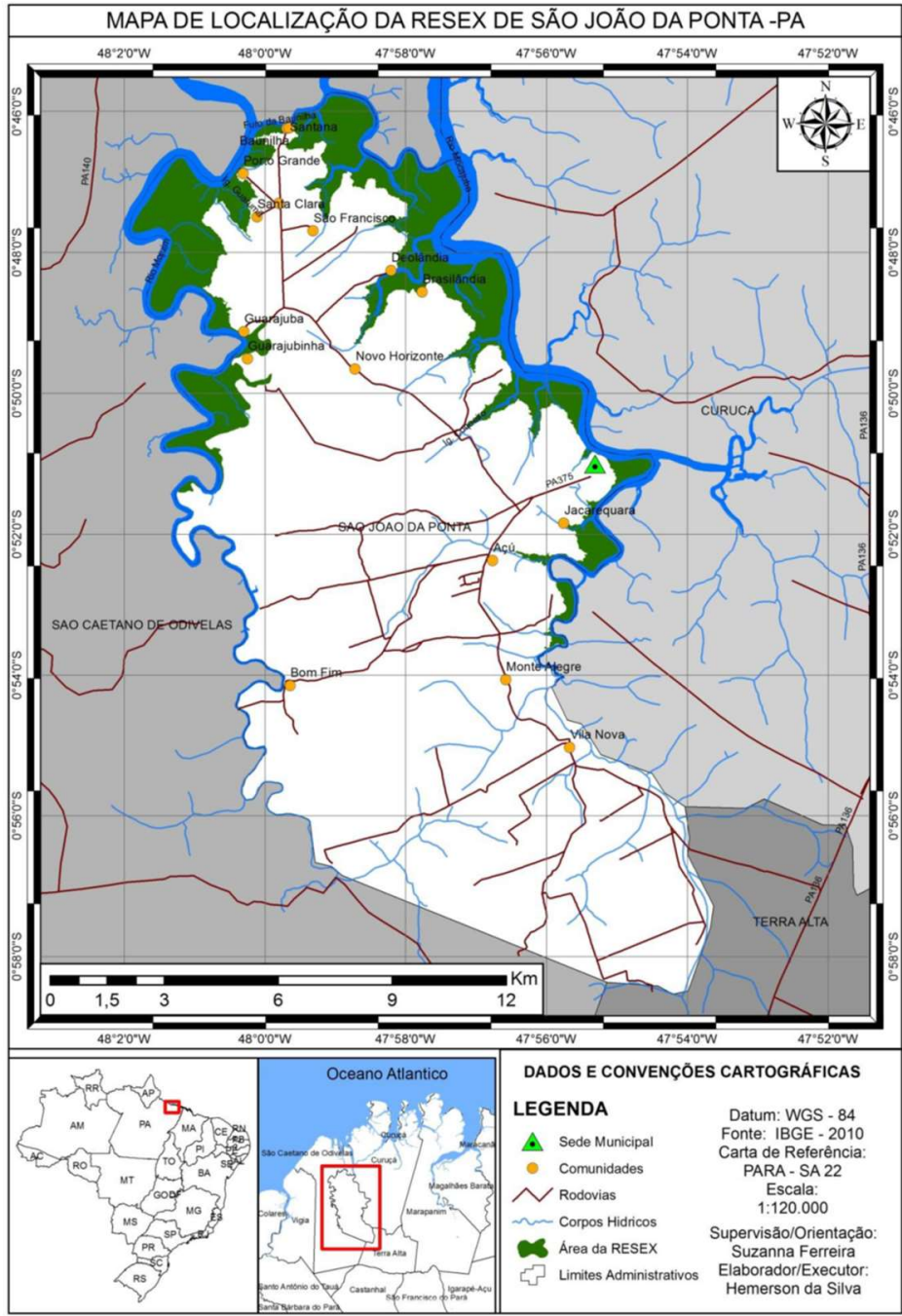

Figura 1: Mapa de localización del Resex São João da Ponta. Fuente: Ferreira (2017).

Considerando los hogares con ingresos mensuales de hasta medio salario mínimo per cápita, el 54,1\% de la población presenta estas condiciones, lo que la coloca en el puesto 28 de 144 entre los municipios del estado y en el puesto 613 de los 5.570 de los municipios de Brasil (IBGE, 2010).

Cuenta con 1 escuela de secundaria, 11 de escuela nivel primaria y 11 y 59 docentes correspondientemente, También con 4 establecimientos de salud SUS (IBGE, 2010).

En el 2010, la tasa de la actividad de la población mayor de 18 años, es de 59,39\%. Además, haciendo un hincapié al desempleo que es representado con un $2.37 \%$, a diferencia de años anteriores como en el 2000 que era un 9,70\% (Atlas de desarrollo humano del - IBGE, 2017). 
Tabla 1: Empleo de la población de 18 años o más - Municipio - São João da Ponta - PA.

\begin{tabular}{|c|c|c|}
\hline Nombre & 2000 & 2010 \\
\hline Tasa de actividad: 18 años o más & 47,47 & 59,39 \\
\hline Tasa de desempleo: 18 años o más & 9,70 & 2,37 \\
\hline Grado de formalidad de las personas empleadas: 18 años o más & 12,38 & 17,57 \\
\hline \multicolumn{3}{|l|}{ Nivel educativo de los empleados } \\
\hline \% de personas empleadas con educación primaria completa - 18 años o más & 24,50 & 38,88 \\
\hline$\%$ de ocupados con bachillerato completo - 18 años o más & 8,85 & 18,85 \\
\hline \multicolumn{3}{|l|}{ Rendimiento medio } \\
\hline \% de personas empleadas con ingresos de hasta $1 \mathrm{sm} \mathrm{-} 18$ años o más & 78,10 & 70,54 \\
\hline \% de personas empleadas con ingresos de hasta $2 \mathrm{sm} \mathrm{-} 18$ años o más & 92,16 & 96,23 \\
\hline \% de personas empleadas con ingresos de hasta $5 \mathrm{sm}-18$ años o más & 97,58 & 99,18 \\
\hline
\end{tabular}

Fuente: PNUD, IPEA y FJP, consultado por IBGE (2017).

Tabla 2: Las actividades económicas del Municipio de São João da Ponta, los siguientes.

\begin{tabular}{lll}
\hline Catastro de empresas & & \\
\hline Titulo & Cantidad & Unidad de medida \\
Central de empresas & 13 & unidades \\
\hline Personal ocupado & 315 & Personas \\
Personal ocupado asalariado & 307 & Personas \\
\hline Salarios y otras remuneraciones & 5842 & X1000 R\$ \\
\hline
\end{tabular}

Fuente: IBGE (2017).

Tabla 3: Censo agropecuario del Municipio São João da Ponta.

\begin{tabular}{|c|c|}
\hline \multicolumn{2}{|l|}{ Censo agropecuario } \\
\hline & Unidad de medida \\
\hline Áreas de establecimientos agropecuarios & establecimientos \\
\hline $\begin{array}{l}\text { Número de establecimientos } \\
\text { agropecuarios }\end{array}$ & establecimientos \\
\hline Agricultura permanente & $\begin{array}{l}\text { Piña, Calabaza, Fresa, Jerimum, arroz, frejol, Yuca (AIPIM, MACAXEIRA), Melón, } \\
\text { Maíz }\end{array}$ \\
\hline Pecuaria & $\begin{array}{l}\text { Bovinos, Caprinos, Equinos, Pollos (Gallos, Pollitos, Gallinas), Mulas, Ovinos, } \\
\text { Cerdos. }\end{array}$ \\
\hline
\end{tabular}

Fuente: IBGE (2017).

Después de la emancipación del municipio de São Caetano de Odivelas, el Municipio de São João da Ponta, en los años 90's, como otras localidades comenzó a preocuparse con las actividades extractivas desde el punto de vista ambiental, proponiendo el manejo de los recursos naturales, ya que años anteriores se estaba viviendo de actividades con la extracción del Caucho, que desde la caída del mismo, los shiringueros se preocupaban en realizar otras actividades como la agricultura y la pesca (CORRÊA, 2016).

Las reservas extractivas de uso sostenible, son áreas destinadas ubicadas en zonas costeras donde la principal característica es la presencia de los manglares, destinados a la extracción de los recursos naturales con una conciencia socio ambiental (FERREIRA, 2013).

La Reserva extractiva de São João da Ponta, fue creada en el 2002, que de acuerdo con el artículo 1 del Decreto S/N del 13 de diciembre del 2002; indica como objetivos la conservación y el uso sostenible de los recursos naturales renovables protegiendo los medios de vida y la cultura de la población extractiva (BRASIL, 2002).

Una de las actividades de la zona es la de extracción de cangrejos en los Manglares de la cuenca del Rio Mocajuba, que mediante reglamentación ambiental hay una demarcación para la actividad ya que también hay zonas para pesca y delimitación con otras comunidades por lo cual existen conflictos; la forma de recolección del recurso natural es en la zona baja de la cuenca (zona de lodo) donde los cangrejos son capturados por la técnica del Lazo o la de la tapa. La técnica del "lazo" consiste en colocar trampas con hilos 
de nylon en lugares estratégicos, y la técnica de la "Tapa" es el impedimento de la salida de los crustáceos de la salida de sus escondrijos con el barro del manglar (ICMBIO, 2010).

También, otra acción para sus sustentabilidad, es la prohibición de la extracción de los cangrejos en las épocas de Veda, en la que coinciden con el tiempo de reproducción y con la liberación de sus huevos (BRASIL, 2020).

La tentativa de este trabajo, se genera a base de una pregunta ¿Cuan desarrollado sosteniblemente está el Municipio de São João da Ponta? Dar un enfoque de desarrollo sostenible aplicado en las comunidades rurales que realicen actividades extractivas con uno de las fuentes económicas principales; por lo cual se llevó a investigar y obtener información con base de trabajos anteriores en la zona de estudio, además, ser de gran aporta para la sociedad.

De acuerdo con el Informe de desarrollo humano, muestra al Brasil en el puesto 79o con un 0.761 de valor (PNUD, 2019). Y es por eso que este estudio tiene el objetivo de evaluar el comportamiento del Municipio de Sao Joao da Ponta, mediante la evaluación del Índice de Desarrollo Sostenible Municipal (IDSM), considerando indicadores que abarcan lo económico, social y medioambiental.

En la obra de Martins et al. (2008), para el caso de los municipios de los Estado de Brasil, considera dimensiones importantes para poder observar el Desarrollo Sostenible, como es el caso de seis puntos: medio ambiente, cultura, demografía, institucional, política y social (MARTINS et al., 2008).

Para este análisis, se realizó la búsqueda correspondiente de los variables e indicadores propuestos en la obra de Martins et al. (2008), por lo cual se pudieron encontrar a disponibilidad de información fidedigna y confiable del año 2010 de los Municipios del Estado de Pará, las fuentes fueron las plataformas web del estado, como es el caso del Atlas de Desarrollo Humano en Brasil (ADHB), Comercio Exterior y Servicios (MDIC / SECEX / DEPL), Instituto Brasileño de Geografía Estadística (IBGE), Matriz de información social (MIS), Portal de salud (DATASUS); Ministerio de Industria, y Sistema Nacional de Información Sanitaria (SNIS).

En el proceso de análisis se puede indicar que los datos tienen la esencia cuantitativa y cualitativa, se utilizó el método de estandarización y medida de la sostenibilidad en la urbe a través del IDSM obtenida de la obra de Martins et al. (2008).

Para este estudio, se consideraron tres dimensiones: Dimensión Social, Dimensión Económico y Dimensión Medio Ambiental, se tuvieron en cuenta estos puntos con la ayuda del "TIPLE BOTTOM LINE", llamada de 3P o 3BL, por lo que es usado para ver la en la que abarca en el tema de la sostenibilidad donde el sector medio ambiental (Planet), social (People) y económico (Profit); son considerados para analizar la huella del carbono, medio ambiental en la sociedad mediante el uso positivo o negativo de los recursos naturales y que ellas es generado mediante las ventas, negocios empleo, etc. (MUKHOPADHYAY et al., 2020).

Entre las variables que forman parte de las tres dimensiones de análisis, se seleccionaron aquellas que tenían cierta disponibilidad de información. Así, la configuración final de los datos utilizados consta de 20 variables, 9 de las cuales son de carácter social, 7 de carácter económico y 4 asociadas al tema ambiental. Así, se recolectaron datos para el municipio de São João da Ponta, además del valor más alto y más bajo entre 
los municipios de Pará para cada variable. Esto se debe a que el método propuesto por Martins et al. (2008) utiliza un procedimiento comparativo entre realidades urbanas en una misma unidad federativa del municipio dirigido a, así, diagnosticar escenarios de sostenibilidad urbana.

Luego, se va realizando la ponderación de 0 a 1; si la coincidencia es positiva, el valor de indicador es directamente proporcional con el IDS; sin embargo, si es negativa, es proporcionalmente inverso al indica (MARTINS et al., 2008), de acuerdo con ello es de vital importancia para la elección de la ecuación alternativa para calcular el IDS para cada variable. Esto se puede obtener comparando los datos de los variables para el caso del municipio de São João da Ponta en relación con otros municipios del estado de Pará.

En el análisis de la sustentabilidad, se hizo la comparación de todos los municipios del Estado de Pará, pero siempre resaltando a São João da Ponta como centro para la investigación; se utilizaron las ecuaciones 1 y 2 de acuerdo con Martins et al. (2008) En relación a cada variable que ha sido verificado del nivel 0 hasta el 1, es decir, mientras más se acerca al cero (0), indica que la situación del municipio es peor; caso contrario los que consiguen tener o llegar hasta el otro extremo con 1 de puntaje, indica que está en buenas condiciones.

$$
\begin{aligned}
& I=\frac{x-m}{M-m} \\
& \boldsymbol{I}=\frac{\boldsymbol{M}-\boldsymbol{x}}{\boldsymbol{M}-\boldsymbol{m}}
\end{aligned}
$$

Ecuación 1 - Cuando en el análisis resulta ser positiva.

Ecuación 2 - Cuando en el análisis resulta ser negativa.

Donde: I = índice calculado para cada variable; $\mathrm{x}=$ Valor de cada variable en el municipio estudiado; $\mathrm{m}=$ Valor mínimo identificado en el estado al que pertenece el municipio; $\mathrm{M}=$ Valor máximo identificado en el estado al que pertenece el municipio.

\begin{tabular}{|c|c|c|}
\hline Índice (0 -1) & Color & Nivel de sustentabilidad \\
\hline $0,0000-0,2500$ & & Critico \\
\hline $0,2501-0,5000$ & & Alerta \\
\hline $0,5000-0,7500$ & & Aceptable \\
\hline $0,7501-1,0000$ & & Ideal \\
\hline
\end{tabular}

Tabla 4: Niveles de sustentabilidad.

Fuente: adaptado de Martins et al. (2008).

En la Tabla 4, se puede tener en cuenta de acuerdo al nivel de la sustentabilidad de la municipalidad a analizar, advirtiendo o felicitando el desarrollo mediante el Índice de Desarrollo Sostenible Municipal (IDSM).

\section{RESULTADOS Y DISCUSIÓN}

De acuerdo con Martins et al. (2008), en su trabajo indican los variables e indicadores para la sostenibilidad en una comunidad rural, en esta oportunidad se trabajaron con los datos encontrados en todas las plataformas virtuales de Brasil; en este caso, se tendrán como punto principal los datos del año 2010, por tener mayor cobertura de información a diferencia de otros años.

Cabe recordar que los sectores que fueron seleccionados para este trabajo son Sociedad (Persons), Economia (Profit) y Medio Ambiente (Planet), para este análisis, que son parte del TIPLE BOTTOM LINE. 
Cuadro 1: Variables e Indicadores de la Dimensión Social para el análisis del IDS de São João da Ponta.

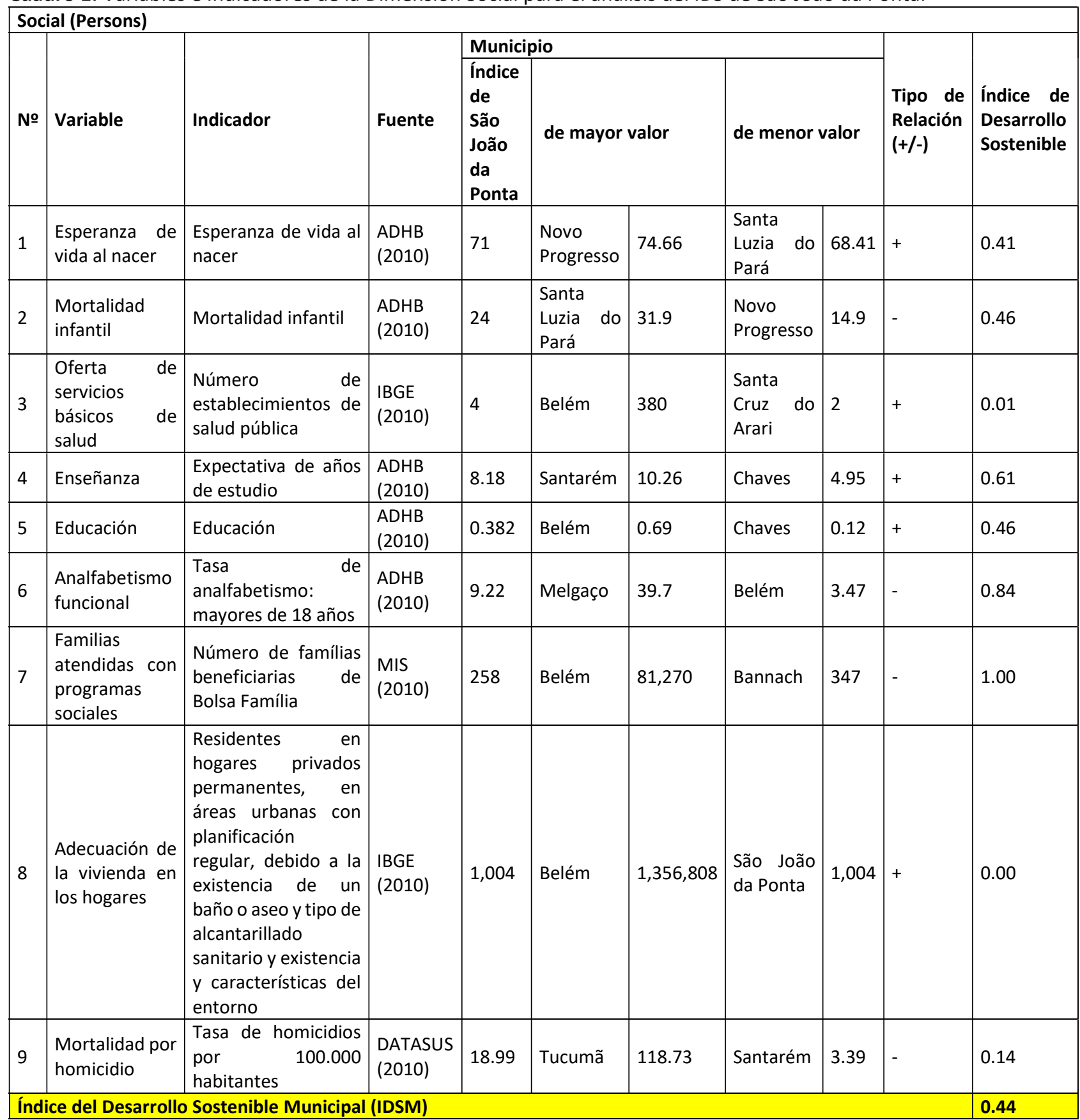

La Dimensión social, abarca temas como seguridad ciudadana, salud, mortalidad, esperanza de vida al nacer, de calidad de vida de la sociedad (MARTINS et al., 2008) del Municipio de São João da Ponta.

De acuerdo con la Cuadro 1, se puede observar una dura batalla entre la expectativa de años de estudio con 0.61 y la Tasa de analfabetismo: mayores de 18 años con 0.84 . Por otro lado, el número de familias beneficiarias de Bolsa Familia cuenta con 1.00. Entonces, para esta Dimensión el Municipio de São João da Ponta, está en alerta con 0.44, dato por el cual que de acuerdo con Pereira et al. (2020), se dista mucho del Municipio de Parauapebas que también es parte del Estado de Pará con 0,65.

En esta Dimensión, está relacionado con el record económico, fuentes de trabajo, Producto Bruto Interno que dicen del crecimiento de una población (MARTINS et al., 2008), en el Municipio de São João da Ponta, estamos en estado Crítico para el año 2010 con 0.19, en la cual se debería tener en cuenta dicho sector y que al comparar con otros municipios como es el caso de Parauapebas que tiene el 0.84 de IDSM y es 
considerado como Ideal (PEREIRA et al., 2020). Aunque, no toda está perdido, ya que con 0.54 el Ingreso familiar mensual nominal per cápita - valor promedio, da una visión de esperanza para la zona.

Cuadro 2: Variables e Indicadores de la Dimensión Económico para el análisis del IDS de São João da Ponta.

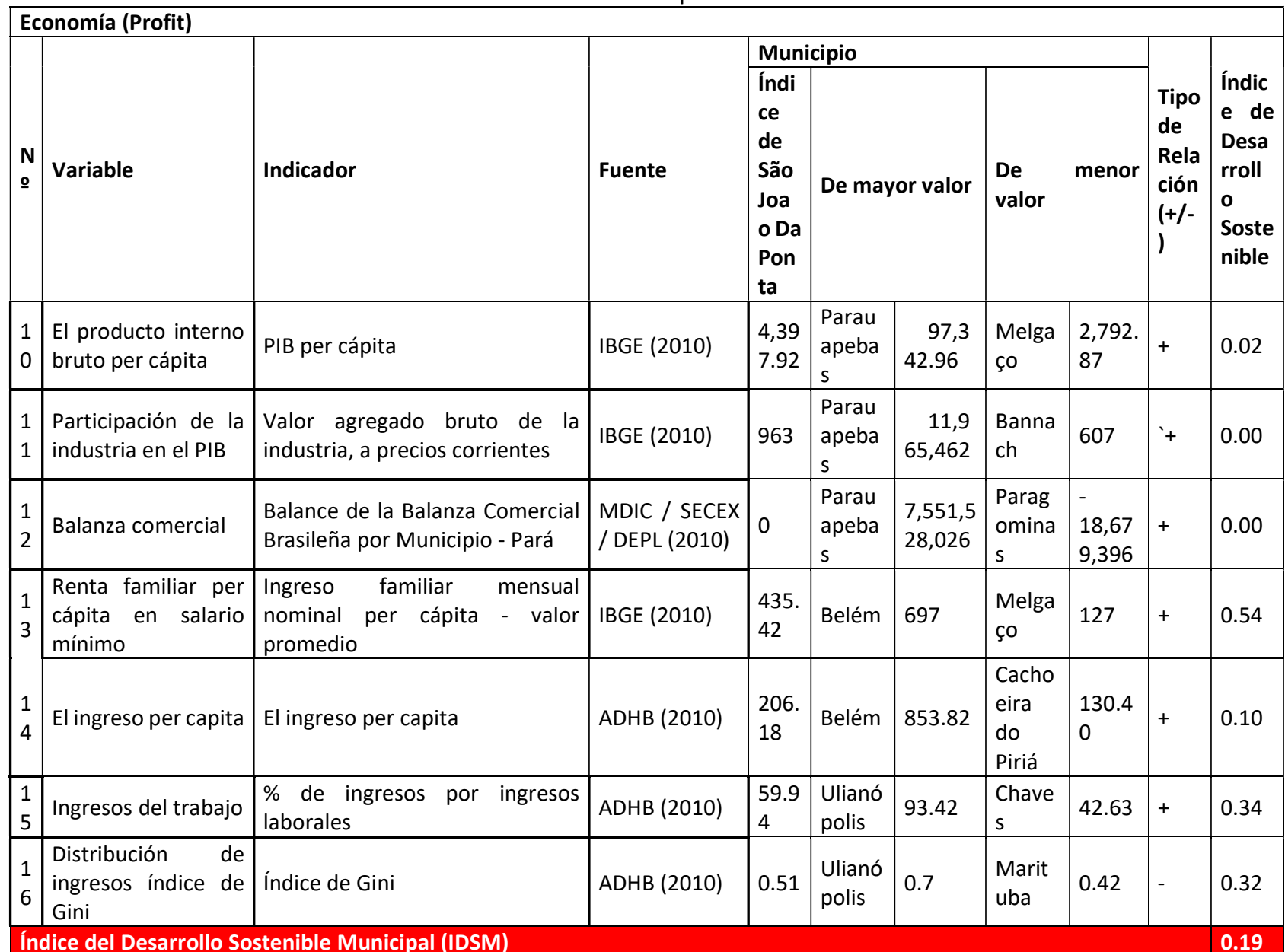

Cuadro 3: Variables e Indicadores de la Dimensión Ambiental para el análisis del IDS de São João da Ponta.

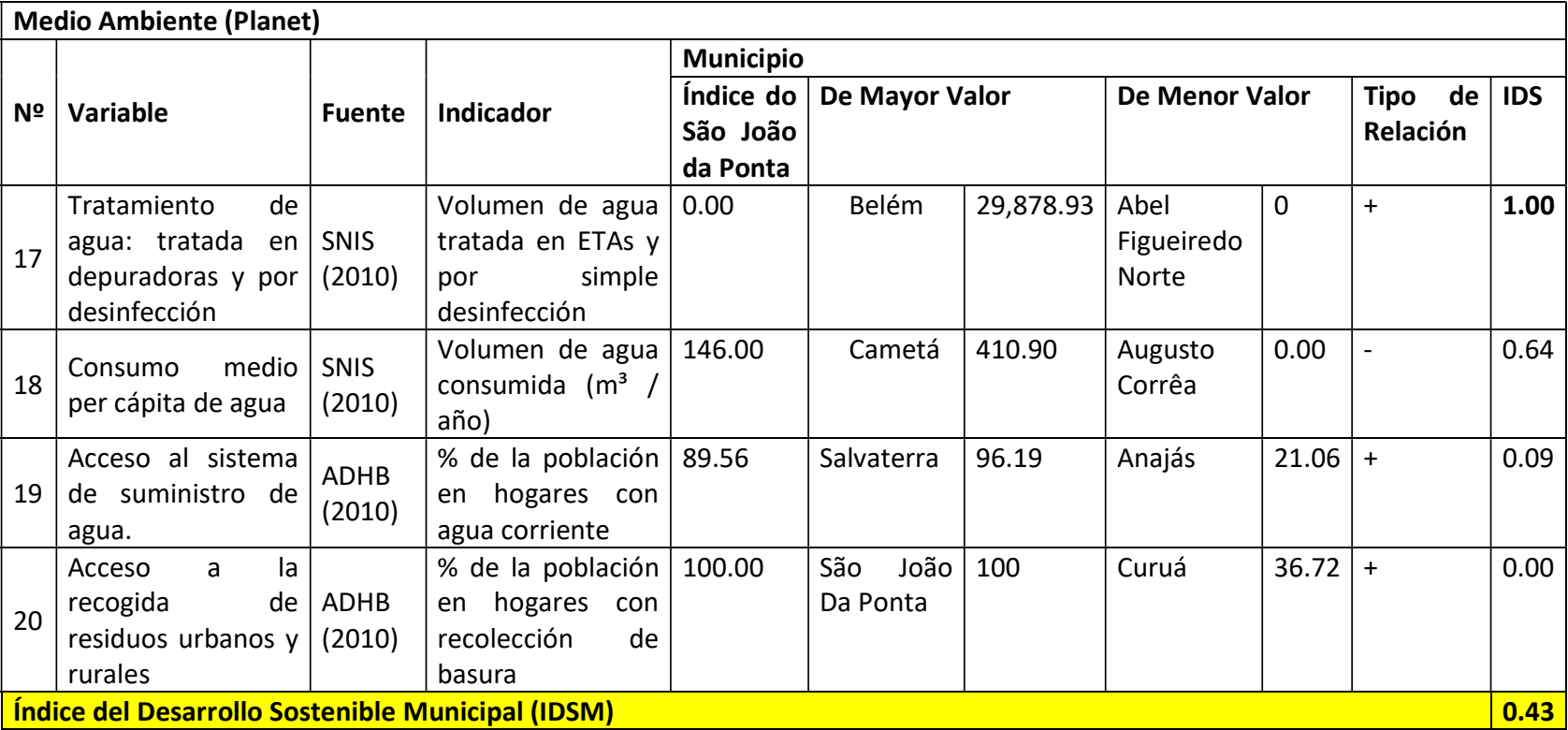

En la Dimensión Medio Ambiental, es considerado los Recursos Naturales, su degradación, protección, cuidados, manejo ambiental que son necesarios para la vida de la población (MARTINS et al., 2008). 
Para el municipio de São João da Ponta, se percibe que hay buenos resultados para el 2010 entre el Volumen de agua tratada en ETAs y por simple desinfección que está con 1.00 y el Consumo medio per cápita de agua con 0.64. Referente al IDSM de la Dimensión es de 0.43, para el Municipio de Parauapebas tiene un IDSM 0.64 aceptable (PEREIRA et al., 2020).

Teniendo en cuenta las tres dimensiones que es parte de la Triple Bottom Line, se puede decir que 0.44 (Dimensión Social), 0.19 (Dimensión Económica) y 0.43 (Dimensión Medio Ambiental) del análisis del Desarrollo Sostenible Municipal (IDSM) de Sao Joao da Ponta, en promedio está con 0.35, esto quiere decir que hay que estar en alerta, mientras se está en crecimiento urbano y en constante mejora.

\section{CONCLUSIONES}

En la Dimensión Social, las variables de expectativa de años de estudio con 0.61 y la Tasa de analfabetismo: mayores de 18 años con 0.84 , muestra la falta implementación de más acciones en el sector educación. En la Dimensión Social, el valor de familiar beneficiarias de Bolsa Familiar es de 1.00, lo cual indica que el Estado está cumpliendo con la realización de programas sociales.

Para el Municipio de São João da Ponta para el año 2010, está en estado de alerta en la Dimensión Social por contar con el valor de 0.44 de IDSM. Para el 2010, en el Municipio de São João da Ponta, cuenta con un valor de 0.54 en el Ingreso familiar mensual nominal per cápita - valor promedio y con 0.34 en el número de ingresos laborales, los datos más altos en la Dimensión Económica.

En general para la Dimensión Económica, el Municipio de São João da Ponta, está en estado Crítico con 0.19 de IDSM para el año 2010; sin embargo, en el Municipio de Parauapebas tiene el 0.84 de IDSM que está en estado Ideal.

Para el municipio de São João da Ponta, se percibe que hay buenos resultados para el 2010 entre el Volumen de agua tratada en ETAs y por simple desinfección que está con 1.00 y el Consumo medio per cápita de agua con 0.64. En la Dimensión del Medio Ambiente para el 2010, el IDSM es de 0.43 eso indica que está en estado de alerta, para el Municipio de Parauapebas, tiene un IDSM 0.64 aceptable.

Teniendo en cuenta las tres dimensiones que es parte de la Triple Bottom Line, se puede decir que 0.44 (Dimensión Social), 0.19 (Dimensión Económica) y 0.43 (Dimensión Medio Ambiental) del análisis del Desarrollo Sostenible Municipal (IDSM) de São João da Ponta, en promedio está con 0.35, esto quiere decir que hay que estar en alerta, mientras se está en crecimiento urbano y en constante mejora.

\section{REFERENCIAS}

BRASIL. Decreto de 13 de dezembro de 2002. Criação da Reserva Extrativista Marinha de São João da Ponta-PA. Brasília: DOU, 2002.

BRASIL. Instituto Brasileiro do Meio Ambiente e dos Recursos Naturais Renováveis. IBAMA Documentos: Período do Defeso. Brasília: IBAMA, 2020.

CASTILLO, T.; BENÍTEZ, L.; PEREIRA, J.. Desarrollo Sostenible Y Evolución De La Legislación Ambiental En Las MIPYMES Del Ecuador. Quito: Universidad Metropolitana, 2017.
CORRÊA, G.. Análise da paisagem na bacia hidrográfica do Rio Mocajuba, Nordeste Paraense, a partir do modelo Teórico GTP. Dissertação (Mestrado) - Universidade Federal do Pará, Belém, 2016.

CRISPIM, D. L.; FERNANDES, L. L.; ALBUQUERQUE, R. L. O. Aplicação de técnica estatística multivariada em indicadores de sustentabilidade nos municípios do Marajó-PA. Revista Principia, v.46, p.145-154, 2019. 
FERREIRA, S. S.. Entre marés e mangues: paisagens territorializadas por pescadores da Resex marinha de São João da Ponta/PA. Dissertação (Mestrado) - Universidade Federal do Pará, Belém, 2017.

FERREIRA, W.. Diagnóstico ambiental da Reserva Extrativista Marinha de São João Da Ponta: Subsídios ao Planejamento Ambiental. Dissertação (Mestrado) Universidade Federal do Pará, Belém, 2013.

GÁMEZ, F.. La dimensión social: un proceso sinérgico en la interacción universidad-comunidad a través de la función de extensión. Caracas: Universidad Pedagógica Experimental Libertador, 2014.

GARAY, C.. Análisis de los avances de los objetivos de desarrollo sostenible-ODS en el municipio de Villa Elisa, departamento central-Paraguay. San Lozenzo: Universidad Nacional de Asunción, 2020.

IBGE. Instituto Brasileiro de Geografía e Estatística. Atlas de desenvolvimento Humano. Censos demográficos, 1991, 2000 e 2010. PNAD Contínua. 2016 e 2017. Brasília: IBGE, 2017.

IBGE. Instituto Brasileiro de Geografía e Estatística. Censo Demográfico de 2010. Rio de Janeiro: IBGE, 2010.
ICMBIO. Instituto Chico Mendes de Conservação da Biodiversidade. Caracterização dos Aspectos Socioambientais e Econômicos da Reserva Extrativista de São João da Ponta e Proposta de Estudos Complementares. Brasilia: ICMBIO, 2010.

KOTZIAN, H. B.; MARQUES, D. M.. Lagoa Mirim e a convenção Ramsar: um modelo para ação transfronteiriça na conservação de recursos hídricos. Revista de gestão de água da América Latina, v.1, n.2, p.101-111, 2004.

MARTINS, M. F.; CÂNDIDO, G. A.. Índice de Desenvolvimento Sustentável para Municípios (IDSM): metodologia para análise e cálculo do IDSM e classificação dos níveis de sustentabilidade: uma aplicação no Estado da Paraíba. João Pessoa: Sebrae, 2008.

MUKHOPADHYAY, B.; MUKHOPADHYAY, B. K.. The Triple Bottom Line. The Sentinel, 2020.

PEREIRA, M. M.; LIMA, G. V. B. A.; CRISPIM, D. L.; FERNANDES, L. L.. Análise de sustentabilidade no município de Parauapebas, Amazônia, Pará, Brasil. Research, Society and Development, v.9, n.3, 2020.

PNUD. Programa das Nações Unidas para o Desenvolvimento. Informe sobre Desarrollo Humano. PNUD, 2019. 